\title{
Effect of Carrying Load on Gait Recognition using J48 with Knee Joint Movements
}

\author{
Jyoti Rana, Nidhi Arora
}

\begin{abstract}
Background: Gait patterns are influenced by various factors. Every person walks differently in different scenarios and it becomes difficult to identify the person correctly by his walking style. Research question: Is it possible to correctly recognize a person through his gait while he walks carrying load? What is the effect of distance on a person's gait while he walks with carrying load? Objective: The paper is an attempt to study the effect of load on gait of a person. As the knee angle varies from person to person in varying conditions, we have studied the effect of load on knee angle and thus on gait recognition. Methods: Experimentation is done on 41 subjects of age group 18-30 years carrying bilateral weights of $2.5 \mathrm{~kg}$ in both hands. Data collected from accelerometer has been studied using $\mathrm{J} 48$ decision tree classification algorithm. Results: Results of experiments shows $86.12 \%$ accuracy in recognizing subjects carrying load up to a distance of 60 meters on a flat surface. The FAR and FRR are found to be $0.77 \%$ and $27.52 \%$ respectively. Conclusion: Carrying load affects the gait of the subject. This makes it difficult to recognize the subject while he walks carrying load. After walking for some distance, the gait pattern and knee angle of the subject shows significant variations.
\end{abstract}

Index Terms: Gait, FAR, FRR, EER, ROC

\section{INTRODUCTION}

Load carriage is common both at domestic places and in offices. Load is carried almost by everyone; by students, postal workers, business workers, shoppers and sports enthusiast etc. Students, business workers and shoppers generally carry school bag or office bag in the form of front pack, back pack and side pack with them while sport enthusiasts carry various sports equipments. Normally these loads vary from $5 \mathrm{~kg}$ to $15 \mathrm{~kg}$ which certainly affects the walking style of the person carrying load. Therefore, the purpose of this work is to study and observe the effect of load carriage on gait of people.

Backpack loading is a significantly different loading condition than double-pack loading with an evenly distributed load. People are mostly observed to carry shopping bags in one or both hands in routine. In this study we have used bilateral load carriage i.e. the load is symmetrically carried either on both shoulders or in both hands. Some previous studies discuss and analyze the influence of subject's gait with carrying load. Goh et al. [6] \& Singh and Koh [7] gave evidence that the body's center of gravity is increased if subject is walking with carrying load and hence walking becomes more unstable.

Revised Manuscript Received on May 20, 2020.

*Correspondence Author

Dr. Jyoti Rana*, Department of Computer Science, Naran Lala College, Navsari, India. Email: jyotirrana@ gmail.com

Dr. Nidhi Arora, SoluSoft Technologies Pvt. Ltd., Ahmedabad, India. Email: nidhig.arora@gmail.com

Martin and Nelson [15] stated in their study that walking pattern of males and females are affected under load conditions. Females require extra steps than adult males due to shorter stride lengths. In their study, Kinoshita and Martin \& Nelson [1-2], found that while carrying a load less than or equal to $50 \%$ of body weight, the duration of the swing phase of gait decreases while the duration of stance phase is not affected. Winsmann and Goldman [3] found that normal walking pattern is significantly modified by either backpack or double-pack external load conditions.

Zarrugh and Radcliffe [4] \& Hughes and Goldman [5] only focused on walking speed and energy cost for gait while carrying load. Majumdar et al. [16] performed a study on load carriage for infantry soldiers. They determined change in speed throughout load carriage even at the preferred self-selected speed of walk which included raise in stride length and cadence. Knee and hip flexion are also affected by load carriage.

To observe the effect of load on the gait and knee angle, data is collected from 41 subjects. The subjects were asked to carry a bilateral fixed weight of $5 \mathrm{~kg}$ for 60 meters on a flat surface. Knee angle of the subjects is studied to understand the effect of load on their gait pattern. The study is done with multiple objectives:

1. To examine the impact of bilateral load on subject's gait while carrying fixed load.

2. To improve the understanding of the effect of load on gait of people who carry heavy load or equipments.

3. To study the impact of knee angle on gait.

4. To examine the effect of distance on subject's gait.

\section{RELATED WORK}

People carry bags of various shapes and weights which may affect their musculoskeletal system possibly leading to pain and spinal lesions. Due to high level of load, person may suffer from the risk of injuries. Bobet and Norman [8] \& Schulze et al. [9] stated that activity of trunk muscles has been affected by load influences. Knapik et al. [17] found that the activity of the trapezius and erector spinae muscles gets affected by load supported via hips and it is mainly found in soldiers. Pascoe et al. [10] concluded in their study that load becomes asymmetrical when the weight of bag increases. It may result into shorten stride length and step length and increase in the contact time of the weight-bearing foot. $\mathrm{Yu}$ et al. [11] stated that gait can be unstable if backpack's weight increases. It may result into increase into step width and decrease in speed and cadence. Study of Son [12] described that bag carrying methods do not influence temporal and spatial gait variable. Yoon [13] studied the correlations between 


\section{Effect of Carrying Load on Gait Recognition using J48 with Knee Joint Movements}

muscle activities and strap length for various types of school bags carried while walking. Gorodnichy \& Hoshino [14] stated that carrying load in terms of back pack, briefcase or laptop in routine is the primary cause of back pain in teenagers. People may have blisters on feet, knee pain, back pain and stress if person carries heavy load for a long time (Martin \& Nelson)[15].

Previous studies [1-5] worked on body weight, duration of swing phase and stance phase, energy cost, walking speed etc. during load carriage, while other studies [6-10] focused on the effect of load on body's center gravity, trunk muscles and symmetric/asymmetric gait. The aim of this work is to introduce a fixed amount of load carriage moment for variable distance to a number of subjects for the purpose of understanding the effect of load on knee joint moments.

\section{GAIT RECOGNITION}

\section{A. Data Collection \& Experimental Setup}

The data collection for experiment is done for 41 healthy and young subjects between age group of 18-30 years. Subjects were asked to carry bilateral fixed weight of $5 \mathrm{~kg}$ and variable distance up to 60 meters. For data collection steps and experimental procedure, please refer [18].

\section{B. Knee Angle}

Limited amount of work has been done on joint kinematics while carrying load. We have calculated knee angle to study the effect of load on subject's gait. Knee angle differs from person to person and it changes in condition like load carriage. Fig. 1 shows angle generated at knee of a subject without and with load. Knee joint angles of all the subjects under the same experimental setup and the same environmental condition are calculated and presented in Table I.

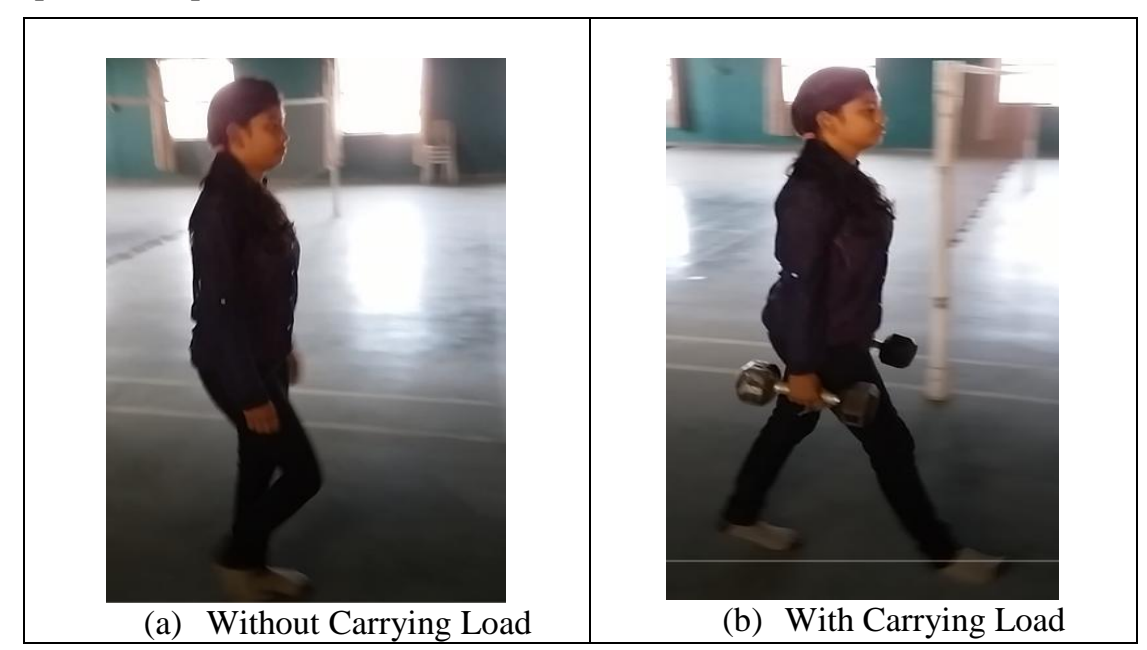

Fig. 1 Knee Angle of Single Subject with and without Load

Table I. Knee Angle of Subjects While Carrying Load

\begin{tabular}{|c|c|c|c|c|c|c|c|c|c|c|c|c|c|c|c|}
\hline Subject Id & 1 & 2 & 3 & 4 & 5 & 6 & 7 & 8 & 9 & 10 & 11 & 12 & 13 & 14 & 15 \\
\hline Knee Angle & 66 & 81 & 80 & 69 & 83 & 85 & 87 & 84 & 85 & 53 & 63 & 89 & 86 & 83 & 81 \\
\hline Subject Id & 16 & 17 & 18 & 19 & 20 & 21 & 22 & 23 & 24 & 25 & 26 & 27 & 28 & 29 & 30 \\
\hline Knee Angle & 83 & 79 & 67 & 88 & 81 & 83 & 72 & 85 & 80 & 44 & 85 & 86 & 86 & 67 & 73 \\
\hline
\end{tabular}

\begin{tabular}{|c|l|l|l|l|l|l|l|l|l|l|l|}
\hline Subject Id & 31 & 32 & 33 & 34 & 35 & 36 & 37 & 38 & 39 & 40 & 41 \\
\hline Knee Angle & 70 & 78 & 78 & 73 & 84 & 72 & 63 & 86 & 89 & 76 & 87 \\
\hline
\end{tabular}

\section{FEATURE EXTRACTION}

The sampling rate and statistical features are extracted. Weka is used for classification of collected data. To measure the performance of distinctive sets of training and testing samples, we have divided original dataset into 70:30 ratios of training and testing datasets. In this study, there are 41 subjects and averages of 2000 records are fetched for each subject. Hence, 92490 total combinations of training and testing data are recorded for this study. A dataset of 92490 samples was divided into two subsets resulting in a training set of 64847 samples and test set of 27643 samples. Table II shows sample dataset. 
Classification accuracy, mean absolute error and root mean

classification accuracy of $86.12 \%$ is achieved. squared error are calculated. By using J48 decision tree,

Table II. Parameters Captured For Subject Walking With Fixed Weight \& Variable Distance

\begin{tabular}{|c|c|c|c|}
\hline $\mathbf{X}$ & $\mathbf{Y}$ & $\mathbf{Z}$ & Sub. \\
\hline-1.407 & 4.453 & 8.446 & $\mathrm{a}$ \\
\hline-1.905 & 4.271 & 7.805 & $\mathrm{a}$ \\
\hline-2.375 & 3.897 & 8.398 & $\mathrm{a}$ \\
\hline-1.254 & 4.127 & 9.998 & $\mathrm{a}$ \\
\hline 1.915 & 3.189 & 9.021 & $\mathrm{a}$ \\
\hline 2.968 & 2.25 & 9.26 & $\mathrm{a}$ \\
\hline 2.25 & 2.47 & 10.63 & $\mathrm{a}$ \\
\hline-0.392 & 2.93 & 11.195 & $\mathrm{a}$ \\
\hline-0.909 & 3.696 & 9.682 & $\mathrm{a}$ \\
\hline-0.44 & 4.089 & 9.078 & $\mathrm{a}$ \\
\hline-2.499 & 5.774 & 3.667 & $\mathrm{am}$ \\
\hline-0.603 & 6.215 & 9.797 & $\mathrm{am}$ \\
\hline 1.742 & 5.612 & 9.442 & $\mathrm{am}$ \\
\hline 0.976 & 5.257 & 8.207 & $\mathrm{am}$ \\
\hline-1.886 & 5.228 & 8.36 & $\mathrm{am}$ \\
\hline-0.296 & 6.579 & 9.461 & $\mathrm{am}$ \\
\hline 1.886 & 7.939 & 9.299 & $\mathrm{am}$ \\
\hline 2.212 & 6.54 & 8.427 & $\mathrm{am}$ \\
\hline 0.651 & 5.43 & 8.801 & $\mathrm{am}$ \\
\hline
\end{tabular}

\begin{tabular}{|c|c|c|c|}
\hline $\mathbf{X}$ & $\mathbf{Y}$ & $\mathbf{Z}$ & Sub. \\
\hline-2.355 & 1.254 & 8.418 & $\mathrm{~b}$ \\
\hline-2.049 & 2.432 & 6.368 & $\mathrm{~b}$ \\
\hline-1.436 & 2.604 & 7.766 & $\mathrm{~b}$ \\
\hline 0.019 & 3.667 & 8.705 & $\mathrm{~b}$ \\
\hline 3.169 & 6.675 & 9.844 & $\mathrm{~b}$ \\
\hline 5.439 & 8.341 & 9.04 & $\mathrm{~b}$ \\
\hline-3.878 & -0.478 & 9.059 & $\mathrm{~b}$ \\
\hline-2.595 & 2.097 & 10.4 & $\mathrm{~b}$ \\
\hline-1.072 & 4.299 & 8.954 & $\mathrm{~b}$ \\
\hline 2.566 & 2.269 & 11.271 & $\mathrm{~b}$ \\
\hline-2.317 & 5.679 & 9.069 & an \\
\hline 0.067 & 6.215 & 4.462 & an \\
\hline-0.497 & 18.981 & 4.491 & an \\
\hline 6.55 & 16.864 & -4.089 & an \\
\hline-0.517 & 11.128 & -2.059 & an \\
\hline-4.721 & 4.558 & 0.124 & an \\
\hline-0.43 & 2.748 & 0.536 & an \\
\hline-1.168 & 3.974 & -0.057 & an \\
\hline-3.112 & 12.076 & 0.833 & an \\
\hline
\end{tabular}

\begin{tabular}{|c|c|c|c|}
\hline $\mathbf{X}$ & $\mathbf{Y}$ & $\mathbf{Z}$ & Sub. \\
\hline-3.141 & 5.353 & 6.081 & $\mathrm{c}$ \\
\hline-1.407 & 6.234 & 10.4 & $\mathrm{c}$ \\
\hline-0.325 & 6.368 & 10.381 & $\mathrm{c}$ \\
\hline-0.076 & 7.24 & 9.758 & $\mathrm{c}$ \\
\hline-0.938 & 6.876 & 6.895 & $\mathrm{c}$ \\
\hline-1.867 & 4.999 & 1.312 & $\mathrm{c}$ \\
\hline 2.355 & 3.629 & -1.197 & $\mathrm{c}$ \\
\hline-5.228 & 4.97 & 11.338 & $\mathrm{c}$ \\
\hline 0.067 & 4.96 & 9.892 & $\mathrm{c}$ \\
\hline-1.656 & 5.899 & 7.584 & $\mathrm{c}$ \\
\hline-0.938 & 9.471 & 7.92 & ao \\
\hline 0.172 & 8.456 & 7.91 & ao \\
\hline 1.628 & 7.163 & 8.283 & ao \\
\hline 3.61 & 12.804 & 7.852 & ao \\
\hline 5.544 & 13.982 & 1.618 & ao \\
\hline 3.591 & 11.233 & 1.063 & ao \\
\hline 1.158 & 8.858 & -0.459 & ao \\
\hline-0.229 & 5.018 & -0.938 & Ao \\
\hline 0.469 & 5.238 & -1.484 & Ao \\
\hline
\end{tabular}

\section{RESULTS AND DISCUSSIONS}

The results of experiment conducted on the data results into data values from which confusion matrix of $41 \times 41$ are generated for each of the scenarios to get more clarity on the classification done by the proposed model. A confusion matrix summarizes the prediction results on a classification algorithm. It shows the way in which your classification model is confused when it makes predictions i.e. it offers idea about accuracy of classification model and varieties of errors it is making. A Sample confusion matrix consisting of 10 subjects is shown in Table III. Every subject is denoted by letter $\mathrm{A}$ to $\mathrm{J}$. The recognized classes are depicted vertically while the reference classes are shown horizontally. By applying J48 decision tree classifier, we achieved accuracy of $86.12 \%$. The experiments conducted resulted into 0.0094 mean absolute error and 0.0687 root mean squared error.

FAR and FRR are calculated for each subject to examine the rate at which a gait pattern accepts or rejects a subject. FAR is the probability that the system incorrectly matches the input pattern to a non-matching template in the database. It measures the percent of invalid inputs that are incorrectly accepted. We found FAR in the range [0, 0.013] which shows that the probability of input data to falsely match a sample template is acceptable.

Table III. Confusion Matrix of the Classification Using J48

\begin{tabular}{|l|l|l|l|l|l|l|l|l|l|l|l|}
\hline & $\mathbf{A}$ & $\mathbf{B}$ & $\mathbf{C}$ & $\mathbf{D}$ & $\mathbf{E}$ & $\mathbf{F}$ & $\mathbf{G}$ & $\mathbf{H}$ & $\mathbf{I}$ & $\mathbf{J}$ & Acc. \\
\hline $\mathbf{A}$ & 1533 & 0 & 0 & 0 & 0 & 0 & 196 & 0 & 0 & 0 & 0.887 \\
\hline $\mathbf{B}$ & 0 & 1553 & 0 & 0 & 0 & 0 & 0 & 0 & 0 & 0 & 1 \\
\hline $\mathbf{C}$ & 0 & 0 & 1758 & 0 & 0 & 0 & 0 & 0 & 0 & 0 & 1 \\
\hline $\mathbf{D}$ & 0 & 0 & 0 & 1774 & 0 & 0 & 0 & 0 & 0 & 0 & 1 \\
\hline $\mathbf{E}$ & 0 & 0 & 0 & 0 & 1451 & 0 & 0 & 0 & 0 & 0 & 1 \\
\hline F & 0 & 0 & 0 & 0 & 0 & 1561 & 0 & 0 & 7 & 0 & 0.996 \\
\hline $\mathbf{G}$ & 490 & 0 & 0 & 0 & 0 & 0 & 940 & 0 & 0 & 0 & 0.657 \\
\hline $\mathbf{H}$ & 0 & 0 & 0 & 0 & 0 & 0 & 0 & 1489 & 0 & 0 & 1 \\
\hline $\mathbf{I}$ & 0 & 0 & 0 & 0 & 0 & 12 & 0 & 0 & 1403 & 0 & 0.992 \\
\hline $\mathbf{J}$ & 0 & 0 & 0 & 0 & 0 & 0 & 0 & 0 & 0 & 1595 & 1 \\
\hline
\end{tabular}


FRR is the probability that the system fails to detect a match between the input pattern and a matching template in the database. It measures the percent of valid inputs that are incorrectly rejected which shows the probability of input data to fail to match its correct template is tolerated up to a maximum limit of 0.47. FAR and FRR can be evaluated as $\mathrm{FAR}=$ Number of false acceptance / Number of imposter attempts

$F R R=$ Number of false rejection / Number of genuine user attempts

A threshold is set by the system either to accept or reject the subject. Equal error rate (EER) is the point where false accept and false reject curves cross i.e. EER=FAR=FRR. It provides an indicator of the system's performance: a lower EER indicates a system with good level of sensitivity and performance. Fig. 2 shows EER curve. We observed that there is no change in gait while carrying fixed load up to 5 meters but beyond this distance, the gait is seen to show variation. This may be because of tiredness introduced due to load. Hence, the recognition rate reduces as compared to normal gait. The FAR and FRR which we found is good as results stated in different studies.

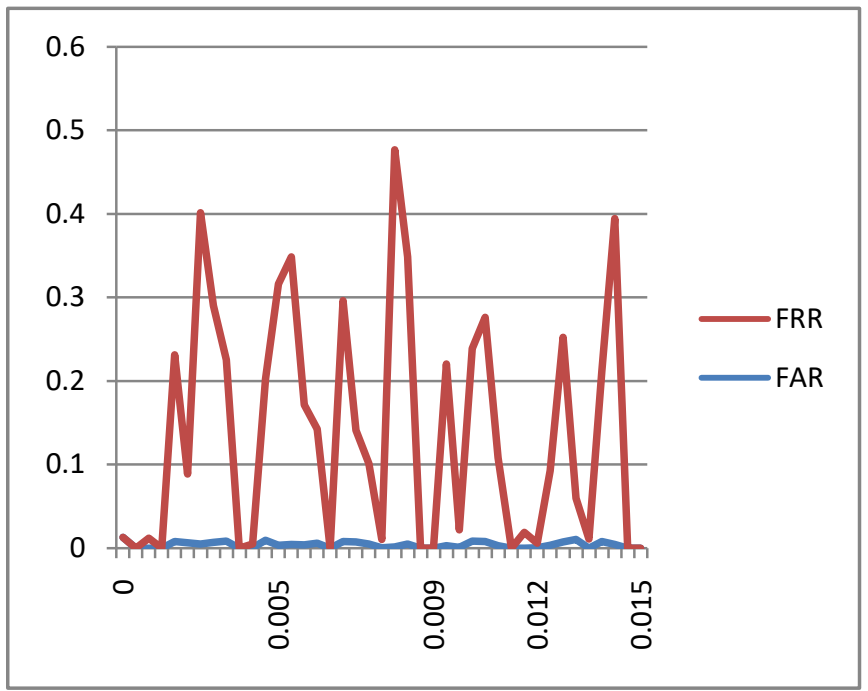

Fig. 2 A EER curve of FAR and FRR

To plot TPR (True Positive Rate) and FPR (False Positive Rate) graphically for every possible threshold values, ROC curve has been generated. To examine the performance of J48 decision tree classifier, ROC curve for a single subject is generated and shown in Fig. 3

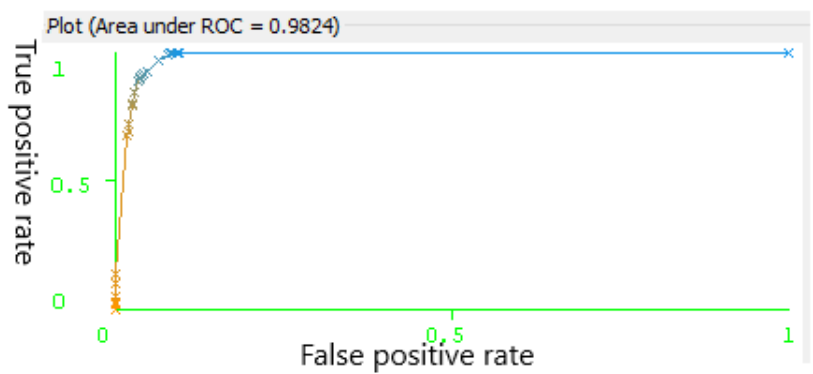

Fig. 3 ROC curve by using J48 Decision tree for a Single Subject

\section{CONCLUSION AND FUTURE WORK}

We have identified subject's gait under varying condition. We have tried to recognize a person using his gait with the help of accelerometer of smart phone while he walks carrying load. The knee angle is found to significant vary for people walking with and without load. Although the changes in knee angle introduce challenges in recognition of the person and after walking for some distance, the gait pattern of the subject shows variation. But, the experiments performed with J48 decision tree classifier, achieved accuracy of $86.12 \%$. The FAR and FRR are found to be $0.77 \%$ and $27.52 \%$ respectively.

We only focused on gait while carrying bilateral fixed weight of $5 \mathrm{~kg}$ and distance up to 60 meters on a flat surface. Future work may include study involving gait recognition while carrying variable weight for some fixed distance. As we know that gait can be influenced by various factors. So it is necessary that each subject must be identified correctly in all scenarios. So that we can find how a system will identify same subject if he walks in varying conditions i.e. with and without carrying load. This will open up many practical implementations of gait biometrics using portable

devices.

\section{ACKNOWLEDGMENT}

We would like to thank all the volunteers for participating in the data collection.

\section{REFERENCES}

1. Kinoshita, H., "Effects of different loads and carrying systems on selected biomechanical parameters describing walking gait," Ergonomics, vol. 28(9), 1985, pp.1347-1362

2. Martin, P. E., and Nelson, R. C., "The effect of carried loads on the combative movement performance of men and women," Military medicine, vol. 150(7), 1985, pp. 357-362.

3. Winsmann, F. R., and Goldman, R. F., "Methods for evaluation of load-carriage systems," Perceptual and Motor Skills, vol. 43(3_suppl), 1976, pp. 1211-1218.

4. Zarrugh, M.Y. and Radcliffe, C.W., "Predicting metabolic cost of level walking," European Journal of Applied Physiology, vol. 38, 1978, pp. 215-223.

5. Hughes, A.L. and Goldman, R.F., "Energy cost of hard work" Journal of Applied Physiology, vol. 29, 1970, pp. 570- 572.

6. Goh, J.H., Thambyah, A. and Bose, K. "Effects of varying backpack loads on peak forces in the lumbosacral spine during walking," Clinical Biomechanics, vol.13, 1988, pp.26-31.

7. Singh, T. and Koh, M., "Effects of backpack load position on spatiotemporal parameters and trunk forward lean," Gait \& Posture, vol. 29, 2009, pp. 49-53.

8. J.Bobet and R.W.Norman, "Effects of load placement on back muscle activity in load carriage," European Journal of Applied Physiology and Occupational Physiology, vol. 53, no. 1, 1984, pp. 71- 75.

9. C. Schulze, T. Lindner, K. Schulz, S. Woitge, W. Mittelmeier, and R. Bader, "Influence of increased load wearing on human posture and muscle activation of trunk and lower limb," Swiss Medical Weekly, vol. 142, supplement 193, 2012, pp. 4-5.

10. Pascoe DD, Pascoe DE, and Wang YT, "Influence of carrying book bags on gait cycle and posture of youths," Ergonomics, vol. 40, 1997, pp. 631-641.

11. Yu JH, Lee DY, and Hong JH, "The effects of a backpack on the ground reaction force in a normal gait," IJACT, vol.5, 2013, pp. 593598.

12. Son S and Noh H, "Gait changes caused by the habits and methods of carrying a handbag," J Phys Ther Sci, vol.25, 2013, pp. 969-971.

13. Yoon JG, "Correlations between muscle activities and strap length and types of school bag during walking," J Phys Ther Sci, vol.26, 2014, pp. 1937-1939. 
14. Gorodnichy, D. O., \& Hoshino, R., "Calibrated confidence scoring for biometric identification," In NIST International Biometric Performance Conference, Vol. 15, 2010, pp. 2-4

15. Martin, P. E., and Nelson, R. C., "The effect of carried loads on the walking patterns of men and women," Ergonomics, vol. 29(10), 1986, pp. 1191-1202.

16. Majumdar, D., Pal, M. S., and Majumdar, D., "Effects of military load carriage on kinematics of gait," Ergonomics, vol. 53(6), 2010, 782-791.

17. J. Knapik, E. Harman, and K. Reynolds, "Load carriage using packs: a review of physiological, biomechanical and medical aspects," Applied Ergonomics, vol.27, no.3, pp.207-216, 1996.

18. Rana, J., Arora, N. and Hiran, D. (2018), "Gait Recognition using J48 Based Identification with Knee Joint Moments," Presented at International Conference on Soft Computing and Signal Processing, Hyderabad, 22-23 June. Advance Intelligent Systems and Computing (AISC): Springer Publication.

\section{AUTHORS PROFILE}

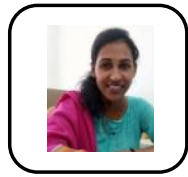

Dr. Jyoti Rana is currently working as an Assistant Professor at Department of Computer Science, Naran Lala College, Navsari affiliated to Veer Narmad South Gujarat University, Surat, India. She is having teaching experience of 10 years. She has published and presented many research papers in international journals. She has also attended many national and international conferences and workshops. Her area of interests are Data and Information Security, Biometrics, Machine Learning and Image Processing.

Dr. Nidhi Arora has more than 19 years of experience in academics, research and industry. She holds doctorate in Neural Networks and has many research papers published in national and international journals. Her research focuses on Artificial Intelligence and its application areas. With publication in IEEE and Springer journals, Dr. Arora is an active member of Editorial board and member of Review board in many international journals. She has been giving her services as reviewer in international conferences held in countries like China, Singapore and Malaysia. 